\title{
Review Article \\ Near-Infrared Spectroscopy for the Evaluation of Anesthetic Depth
}

\author{
Gabriela Hernandez-Meza, ${ }^{1}$ Meltem Izzetoglu, ${ }^{1}$ Mary Osbakken, \\ Michael Green, ${ }^{2}$ and Kurtulus Izzetoglu ${ }^{1}$ \\ ${ }^{1}$ School of Biomedical Engineering, Science and Health Systems, Drexel University, 3508 Market Street, Suite 100, Philadelphia, \\ PA 19104, USA \\ ${ }^{2}$ Department of Anesthesiology, Drexel University College of Medicine, Hahnemann University Hospital, 245 N. 15th Street, \\ MS 310, Philadelphia, PA 19102, USA
}

Correspondence should be addressed to Gabriela Hernandez-Meza; gh88@drexel.edu

Received 24 July 2015; Accepted 23 August 2015

Academic Editor: Paola Aceto

Copyright (C) 2015 Gabriela Hernandez-Meza et al. This is an open access article distributed under the Creative Commons Attribution License, which permits unrestricted use, distribution, and reproduction in any medium, provided the original work is properly cited.

\begin{abstract}
The standard-of-care guidelines published by the American Society of Anesthesiologists (ASA) recommend monitoring of pulse oximetry, blood pressure, heart rate, and end tidal $\mathrm{CO}_{2}$ during the use of anesthesia and sedation. This information can help to identify adverse events that may occur during procedures. However, these parameters are not specific to the effects of anesthetics or sedatives, and therefore they offer little, to no, real time information regarding the effects of those agents and do not give the clinician the lead-time necessary to prevent patient "awareness." Since no "gold-standard" method is available to continuously, reliably, and effectively monitor the effects of sedatives and anesthetics, such a method is greatly needed. Investigation of the use of functional near-infrared spectroscopy (fNIRS) as a method for anesthesia or sedation monitoring and for the assessment of the effects of various anesthetic drugs on cerebral oxygenation has started to be conducted. The objective of this paper is to provide a thorough review of the currently available published scientific studies regarding the use of fNIRS in the fields of anesthesia and sedation monitoring, comment on their findings, and discuss the future work required for the translation of this technology to the clinical setting.
\end{abstract}

\section{Introduction}

In the United States, awareness with recall during general anesthesia has been estimated to occur in 1-2 cases per 1000 patients annually, which amounts to approximately 26,000 cases per year [1-3]. The classifications of events that constitute awareness are somewhat controversial and can range from feelings of pain to recall of conversations during surgery. In other countries, such as China, the incidence of anesthesia awareness has been found to be as much as two times higher than that of the United States [4], while a British audit found the incidence to be lower [5]. The studies conducted thus far have linked awareness with recall to age, female sex, obesity, anesthetist's experience, length of surgery, and previous awareness events. Patients who have suffered from intraoperative awareness have been known to experience long term psychological consequences, most notably posttraumatic stress disorder [6].

Anesthetic agents used during general anesthesia disrupt the activity of neurons in a dose dependent manner in order to suppress memory formation and awareness. This decrease in neural activity can be visualized with positron emission tomography (PET) as a decrease of the glucose metabolic rate (GMR) and the cerebral metabolic rate of oxygen $\left(\mathrm{CMRO}_{2}\right)$ consumption [7-9]. Furthermore, it is to be noted that all volatile anesthetics suppress the cerebral metabolic rate and affect the cerebral blood flow in a dose dependent manner [10]. This information is very important, due to the fact that the cortical regions of the brain, including the prefrontal cortex, experience functional inhibition during anesthesia [11-14]. 
Under the current standard of care published by the ASA, continuous monitoring of oxygenation, ventilation, circulation, and temperature is required [15]. These measurements allow the anesthesia provider to detect adverse events and improve patient safety during surgery; however, they are not specific to the effect of the anesthetics on the brain. The lack of a "gold-standard" method for assessing the effects of anesthetics and sedatives on the brain has led to the investigation of electroencephalographic (EEG) signals, auditory evoked potential (AEP), and facial electromyography (EMG) signals as possible monitors of depth of anesthesia. Currently, devices based on these technologies, such as the Bispectral Index (BIS) monitor (Medtronic, Dublin, Ireland), are clinically available. The BIS monitor evaluates the Fourier domain of electroencephalograph epochs and through a proprietary algorithm outputs a number between 0 and 100, with values under 60 representing the anesthetized state. Still, although accessible, the routine use of EEG monitors has been estimated to be approximately $1.8 \%$ [16]. The low rate of use of these devices may be influenced by reduced performance of the devices in the presence of opioids [17] and the fact that they have been found to provide information that correlates with the patient's state but are not predictive enough to guide the delivery of anesthetics $[18,19]$. In contrast to other means of monitoring depth of anesthesia, near-infrared spectroscopy (NIRS) monitors change in circulatory oxygenation of the cerebral cortex, which can reflect tissue oxygen use $[20,21]$. These facts guided the reasoning for investigating the sensitivity of hemodynamic response measured by functional near-infrared spectroscopy (fNIRS) on the prefrontal cortex (PFC) to determine changes in the anesthetic depth.

fNIRS is a safe, noninvasive, and portable optical method that can be used to monitor activity within the cortical areas of the human brain. Making use of specific wavelengths of light, fNIRS provides measurements of oxygenated $\left(\mathrm{HbO}_{2}\right)$ and deoxygenated $(\mathrm{Hb})$ hemoglobin that are in direct relation with hemodynamic changes in the brain [20-22]. A close link between hemodynamics and neural activity has been established through brain-energy metabolism research [23-25]. Previous studies comparing fNIRS to established neuroimaging technologies, such as functional magnetic resonance imaging (fMRI) and PET, have shown that fNIRS provides comparable results in various domains including attention, memory, and sensory/motor areas in healthy and diseased/disabled populations of various age groups [26-30].

fNIRS has also been used to detect cerebral hypoxia during carotid endarterectomy $[31,32]$ and to monitor cerebral perfusion during liver transplantation [33]. Its capacity to determine the hemodynamic changes that occur in the cerebral cortex as a consequence of anesthetic use makes this technology an ideal candidate for the study and development of monitors for anesthetic depth. Furthermore, because it is portable and noninvasive, fNIRS can be of substantial practical use in clinical settings.

The primary purpose of this paper is to provide a review of studies of anesthesia monitoring using near-infrared spectroscopy to provide a better understanding of the relationship between measurable hemodynamic changes in the prefrontal cortex and changes in anesthetic state. This review also presents information on the challenges associated with the translation of this technology into a clinical environment. This review will begin by outlining the results of investigations of anesthetic drug effects on cerebral metabolism and hemodynamics, continue with studies that evidence potential confounding factors for the fNIRS signal, and conclude with studies that have uncovered potential biomarkers in the fNIRS signal that could signal changes in anesthetic depth.

\section{Methods}

The study selection was performed using the PubMed database to find studies that investigated brain hemodynamics during anesthesia or sedation using functional nearinfrared spectroscopy. The search was restricted to English language articles published between January 1, 1990, and May 1,2015 . The search terms included "functional near infrared spectroscopy," "fNIR," "NIRS," "anesthesia," "sedation," and "neuromarker." During the search, journal articles with topics related to oxygen saturation monitoring during cardiac or other surgeries were excluded. The results of the studies were divided into three groups: (i) findings on the effects of anesthetics on cerebral hemodynamics, (ii) findings related to confounding factors of the fNIRS signal, and (iii) findings on fNIRS biomarkers of depth of anesthesia. Tables 1-3 contain a summary of the findings of the studies presented in this paper.

To further inform the reader on the topics examined in this review we present representative example plots from our data obtained during a study of 50 patients undergoing elective surgery at the Hahnemann University Hospital, Philadelphia, PA. The study protocol and statements of informed consent were approved by the Institutional Review Board (IRB) of Drexel University. The study was conducted with the subjects' understanding and consent. The figures presented in this review originated from $\mathrm{fNIRS}$ data recorded during three elective surgical procedures, of patients classified as ASA class I or II, under general anesthesia using propofol for induction and sevoflurane for maintenance. In this review, data from this study was used to serve as examples, when relevant, for the results described in previous studies.

\section{Results}

The results of the literature search were grouped by the following topics: (i) findings on the effects of anesthetics on cerebral hemodynamics, (ii) findings related to confounding factors of the fNIRS signal, and (iii) findings on fNIRS biomarkers of depth of anesthesia.

3.1. Effects of Anesthetics on Cerebral Hemodynamics. Initial studies of fNIRS in the area of anesthesia examined primarily the differences in cerebral metabolism and hemodynamics elicited by different drugs on the prefrontal cortex. These investigations focused on examining cerebral oxygen saturation $\left(\mathrm{rSO}_{2}\right)$, cerebral blood volume as approximated by the total hemoglobin concentration (HbTot), and cerebral blood flow $(\mathrm{CBF})$ in response to the administration of anesthetics. 


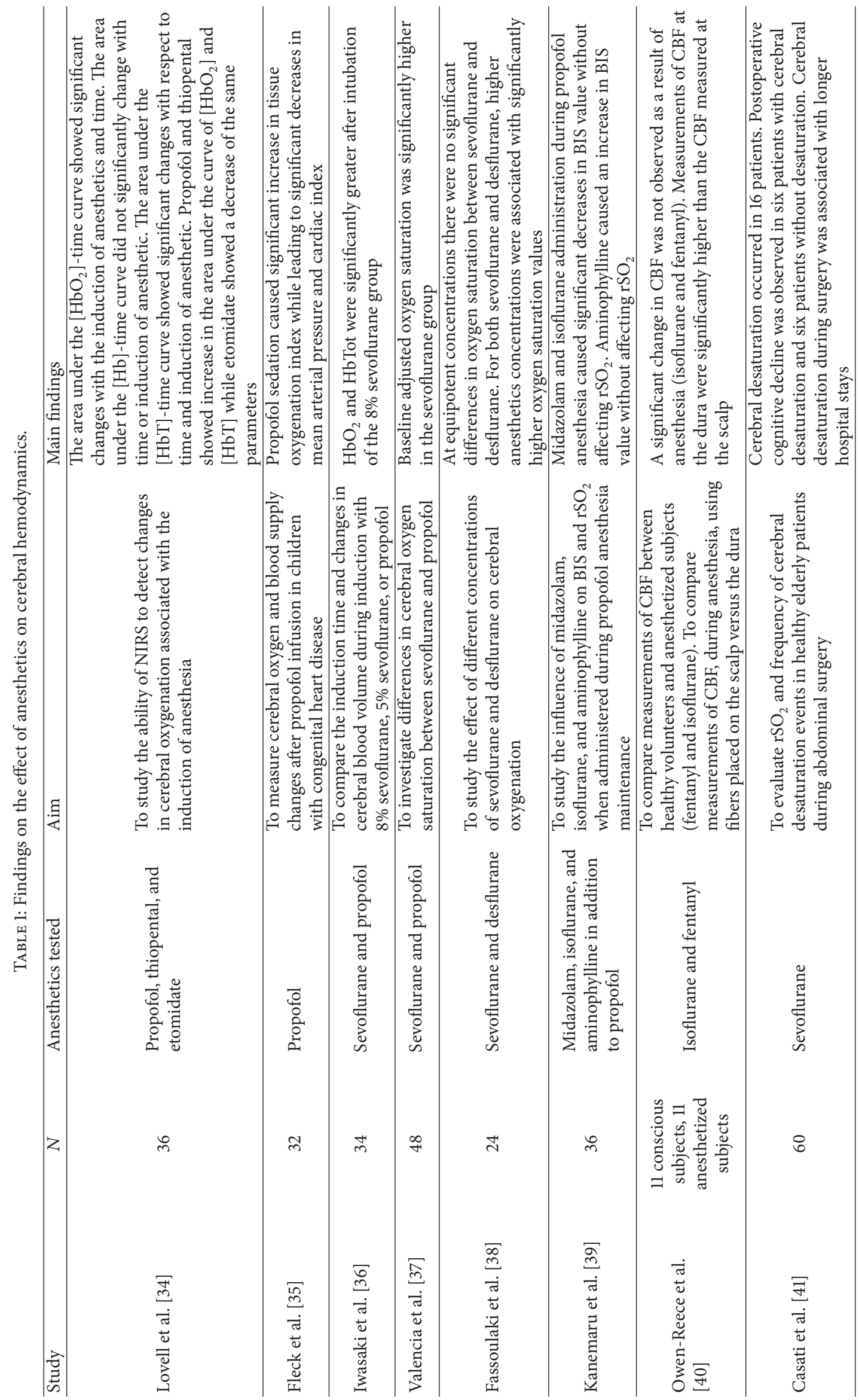




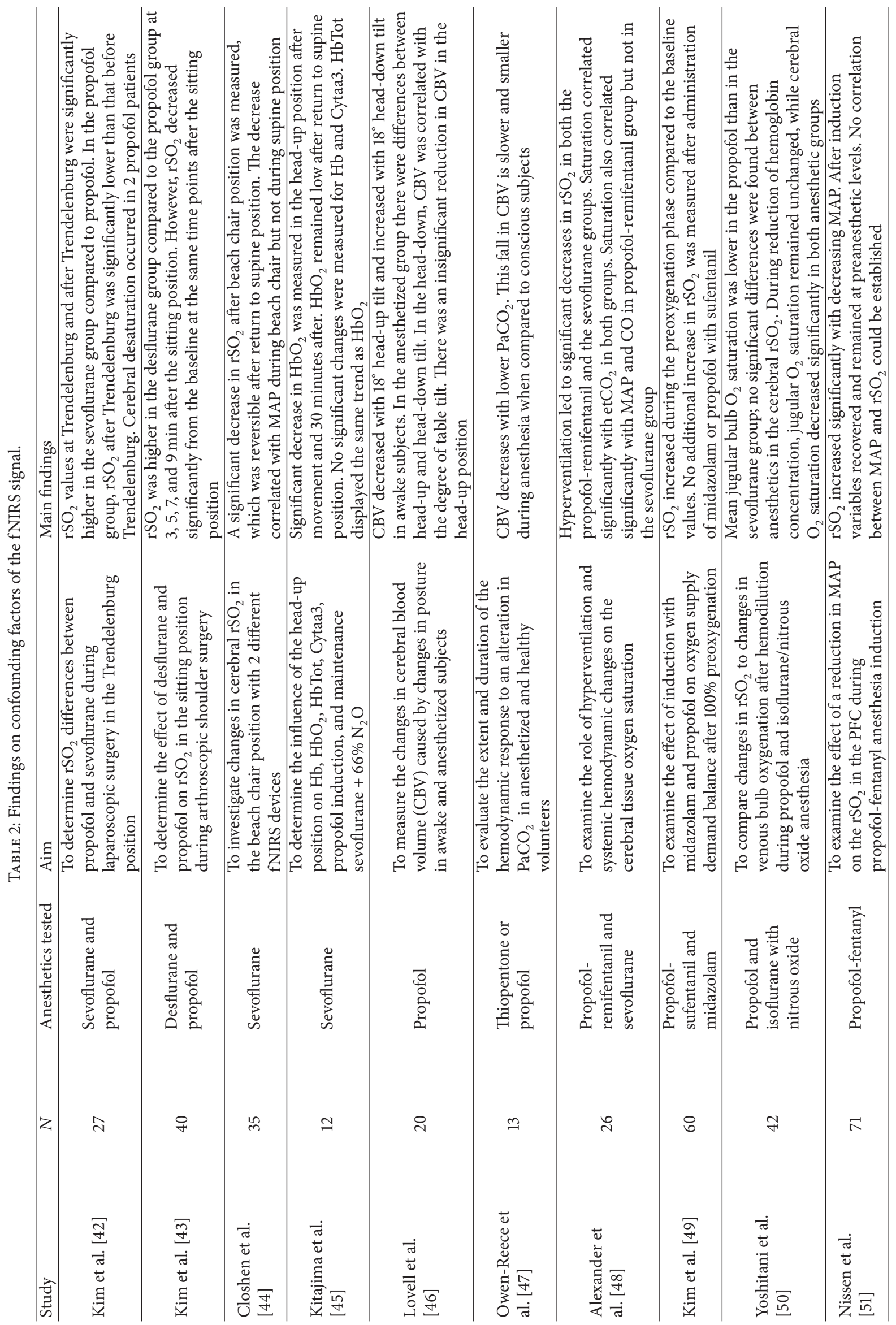


TABLE 3: Findings on fNIRS biomarkers of depth of anesthesia.

\begin{tabular}{|c|c|c|c|c|}
\hline Study & $N$ & Anesthetics tested & Aim & Main findings \\
\hline Izzetoglu et al. [52] & 26 & $\begin{array}{l}\text { Sevoflurane and } \\
\text { desflurane }\end{array}$ & $\begin{array}{l}\text { To determine } \\
\text { neuromarkers that can } \\
\text { differentiate between light } \\
\text { and deep anesthesia }\end{array}$ & $\begin{array}{l}\text { Found a significant difference in Hb between } \\
\text { deep and light anesthetic stages. The mean } \\
\text { values indicate that light anesthesia is } \\
\text { associated with lower Hb. The effect was seen } \\
\text { most predominantly in the right hemisphere. } \\
\text { Deep anesthesia was also associated with a slow } \\
\text { rate of change in Hb, whereas light anesthesia } \\
\text { was associated with a high rate of change }\end{array}$ \\
\hline $\begin{array}{l}\text { Leon-Dominguez et al. } \\
\text { [53] }\end{array}$ & 20 & $\begin{array}{l}\text { Propofol and } \\
\text { sevoflurane }\end{array}$ & $\begin{array}{l}\text { To examine the } \\
\text { contribution of the human } \\
\text { prefrontal cortex to the } \\
\text { emergence and suppression } \\
\text { of consciousness }\end{array}$ & $\begin{array}{l}\text { Propofol causes a significant increase in } \mathrm{Hb} \\
\text { after induction in the left and right PFC. } \\
\text { Removal of sevoflurane during emergence } \\
\text { causes a significant decrease in the levels of } \mathrm{Hb} \\
\text { in the right PFC }\end{array}$ \\
\hline Curtin et al. [54] & 41 & Propofol & $\begin{array}{l}\text { To provide an initial } \\
\text { evaluation into the benefits } \\
\text { of fNIR for the monitoring } \\
\text { of patients during GI } \\
\text { endoscopy }\end{array}$ & $\begin{array}{l}\text { fNIR can detect a dose dependent response to } \\
\text { the infusion of propofol during a GI sedation } \\
\text { regime. Significant increases were found in } \\
\mathrm{HbO}_{2} \text { concentration following propofol } \\
\text { administration }\end{array}$ \\
\hline
\end{tabular}

For clarity, this review separates the studies into two categories: (i) evaluation of fNIRS data during induction and (ii) evaluation of fNIRS data during steady state anesthesia maintenance. A summary of the findings of studies included in this section can be found in Table 1.

3.1.1. Effect of Anesthesia Induction. Anesthetic drugs are known to decrease the $\mathrm{CMRO}_{2}$ and to have a drug and dose dependent effect on CBF. The examination of fNIRS derived hemodynamic changes during the induction of anesthesia provides a method for examining the effects of different drugs during the transition between the awake and anesthetized state.

Using fNIRS, Lovell et al. documented small changes in cortical brain oxygenation during the induction of anesthesia with propofol, thiopental, or etomidate by following the adult patient's hemodynamic changes from a baseline period until 3 minutes after the administration of the intravenous (IV) drugs [34]. Changes in oxygenation were reported as differences in the relative $\mathrm{HbO}_{2}$ and $\mathrm{HbT}$ Tot concentrations. This study found that propofol and thiopental induce an increase in both $\mathrm{HbO}_{2}$ and $\mathrm{HbT}$ Tot relative to the baseline period [34]. In our 50-patient study, we have observed similar changes in $\mathrm{HbO}_{2}$ and $\mathrm{HbT}$ tot following propofol induction. Figure 1 shows an example of the changes in oxygenation that occur after induction of anesthesia with propofol. In contrast, etomidate exhibited a decrease in both $\mathrm{HbO}_{2}$ and HbTot parameters, possibly due to the different effect of this drug on oxygen saturation and blood flow [34]. This study revealed the capacity of fNIRS to measure drug dependent changes in oxygenation associated with anesthetic induction. A similar study on a pediatric population examined the effects of propofol induction on cerebral oxygenation of the frontal cortex [35]. As in the study by Fleck et al., propofol was found to produce an increase in $\mathrm{HbO}_{2}$ and $\mathrm{HbT}$ Tot relative to the conscious period [35].

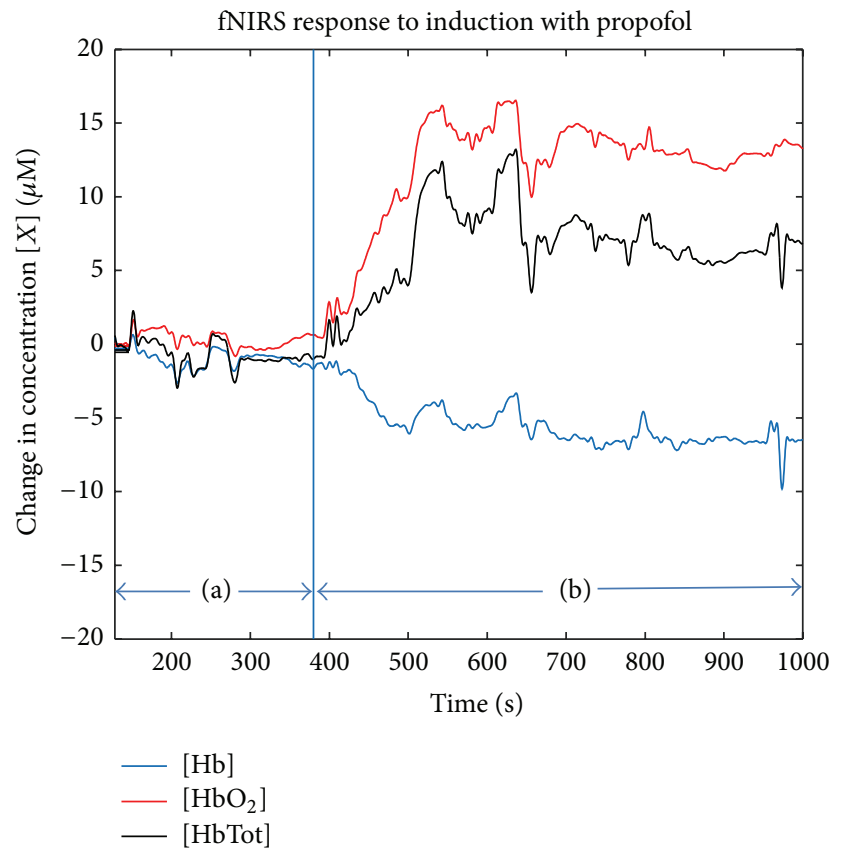

Figure 1: Effect of bolus propofol induction (200 mg) on [Hb], $\left[\mathrm{HbO}_{2}\right]$, and [HbTot]. (a) Time before induction and (b) time after induction. Data collected from a 31-year-old female patient undergoing a laparoscopic hysterectomy. (G. Hernandez-Meza, personal communications, July 22, 2015, School of Biomedical Engineering, Science and Health Systems at Drexel University, Philadelphia, PA 19104).

Iwasaki et al. examined the changes in $\mathrm{HbO}_{2}$ and $\mathrm{HbT}$ tot that occur after induction with $67 \% \mathrm{~N}_{2} \mathrm{O}$ and propofol, sevoflurane at $5 \%$, or sevoflurane at $8 \%$ [36]. The patient's hemodynamic changes were continuously recorded from preinduction period until three minutes after intubation. This study reported significant increases in $\mathrm{HbO}_{2}$ and $\mathrm{HbTot}$ after 
$8 \%$ sevoflurane induction compared to $5 \%$ sevoflurane and propofol [36]. This suggests that fNIRS is sensitive to changes caused by different concentrations of the same anesthetic. This is evidenced by the difference seen between $5 \%$ and $8 \%$ sevoflurane. However, this study does not match the findings of the other propofol induction studies outlined above. The results could be explained by the use of $\mathrm{N}_{2} \mathrm{O}$ in conjunction with propofol during the induction procedure, which differs from the method employed in the studies by Lovell et al. and Fleck et al.

These initial studies provided the ground work to demonstrate that fNIRS is capable of monitoring real time changes that occur as a consequence of the administration of anesthetics. Additionally, it was shown that this technology is capable of distinguishing between the effects of induction with different drugs, such as propofol, etomidate, and thiopental [34]. Different anesthetic concentrations of the same drug, such as $5 \%$ versus $8 \%$ sevoflurane, also registered different fNIRS signal signatures during induction [36].

3.1.2. Evaluations during Anesthesia Maintenance. Initial studies of fNIRS parameters during maintenance focused on examining differences in $\mathrm{rSO}_{2}$ and $\mathrm{CBF}$ between anesthetics to understand the effect of anesthetics on the oxygen supply demand balance and metabolism. Differences in oxygen saturation on the frontal cortex were found between sevoflurane and propofol during maintenance of anesthesia [37]. Sevoflurane was found to produce higher $\mathrm{rSO}_{2}$ values, which indicated that sevoflurane at $1.5 \%$ maintains the $\mathrm{CBF} / \mathrm{CMRO}_{2}$ coupling to a similar extent as propofol [37]. Measurements of $\mathrm{rSO}_{2}$ have also been used to evaluate the incidence of cerebral desaturation events during surgical procedures [37, 41]. An investigation of such events in adults between the ages of 18 and 65 during propofol and sevoflurane anesthesia found that desaturation occurred only during the use of propofol [37]. On the other hand, during a sevoflurane study of elderly patients cerebral desaturation occurred at $26 \%$ of the examined cases [41].

A study by Fassoulaki et al. [38] examined differences on $\mathrm{rSO}_{2}$ between sevoflurane and desflurane during maintenance. First, $\mathrm{rSO}_{2}$ was examined during maintenance with BIS values between 40 and 50 . No difference in oxygen saturation was found between the inhalants desflurane and sevoflurane [38]. Next, $\mathrm{rSO}_{2}$ was measured during maintenance of BIS values between 20 and 30 by increasing the concentrations of the inhalants. Again, no significant difference in $\mathrm{rSO}_{2}$ between inhalants was found [38]. However, this study found that $\mathrm{rSO}_{2}$ was higher when BIS values were maintained between 20 and 30 than the ones obtained when BIS values were kept between 40 and 50 [38]. During the 15 minutes of the measurement at steady state maintenance, the $\mathrm{rSO}_{2}$ level was stable [38]. The study by Fassoulaki et al. [38] provided evidence that $\mathrm{rSO}_{2}$ changes with increasing concentrations of sevoflurane and desflurane.

Kanemaru et al. [39] examined the effect of administration of midazolam, isoflurane, or aminophylline during maintenance of anesthesia with propofol. The goal of this study was to determine if the BIS index changes that occur with additional drug infusion are mirrored by changes in oxygenation. It was found that the three previously mentioned drugs affected the BIS index but not $\mathrm{rSO}_{2}$, suggesting that they may only have a negligible effect on oxygenation [39].

fNIRS has also been employed to examine cerebral blood flow during anesthesia maintenance. A study quantifying CBF during steady state anesthesia maintenance with isoflurane versus conscious patients showed no difference between the two states [40].

The previously outlined studies offer evidence for the capacity of fNIRS to measure differences in oxygenation between types and concentrations of anesthetics that could help in the assessment of anesthetic depth [37, 38]. Moreover, the studies demonstrated that some anesthetic drugs (midazolam, aminophylline, and isoflurane) may affect anesthetic depth, while having no effect on oxygen saturation [39]. To determine the effects of midazolam, aminophylline, and isoflurane on brain oxygenation an investigation of all the fNIRS derived measures (i.e., $\mathrm{Hb}, \mathrm{HbO}_{2}$, and $\mathrm{HbTot}$ ) is necessary. Additionally, the studies indicate that oxygenation remains constant when the depth of anesthesia is maintained at a steady state [38].

3.2. Findings on Confounding Factors of the fNIRS Signal. Several factors can affect the oxygen supply demand balance during fNIRS measurements of patients under anesthesia, affecting the interpretation of the hemodynamic variables recorded by the device. It is important to understand how the fNIRS signal can be affected by these confounding factors so that algorithms can be developed to suppress the effect of confounding information. These factors can be related to the position, type of surgery, hematocrit levels, and ventilation. A summary of the findings of studies included in this section can be found in Table 2 .

3.2.1. Effects of Position. Position change, such as various tilt angles of the operating table, has been demonstrated to affect the hemodynamic parameters measured by fNIRS [42-46]. The hemodynamic changes observed after position change may be related to changes in $\mathrm{CBV}$ associated with the effect of gravity but are also dependent on the anesthetic. Changing to the Trendelenburg position after $\mathrm{CO}_{2}$ pneumoperitoneum was evaluated during propofol and sevoflurane anesthesia. Significant increase in $\mathrm{rSO}_{2}$ after $\mathrm{CO}_{2}$ insufflation and position change was present with the use of sevoflurane but not propofol [42]. This study also found lower $\mathrm{rSO}_{2}$ values for propofol anesthesia [42].

Changing to the sitting position was evaluated during anesthesia maintenance with propofol and desflurane anesthesia [43]. Upon shifting to the sitting position, both groups experienced a significant decrease in $\mathrm{rSO}_{2}$. The propofol group had significantly lower $\mathrm{rSO}_{2}$ values [43]. Oxygen saturation was measured during nine minutes in the sitting position and during this time no significant differences were observed within the groups. Returning to supine position restored $\mathrm{rSO}_{2}$ values to the baseline level [43]. The results show that moving to the sitting position caused a decrease in cerebral oxygen saturation in equipotent concentrations of 


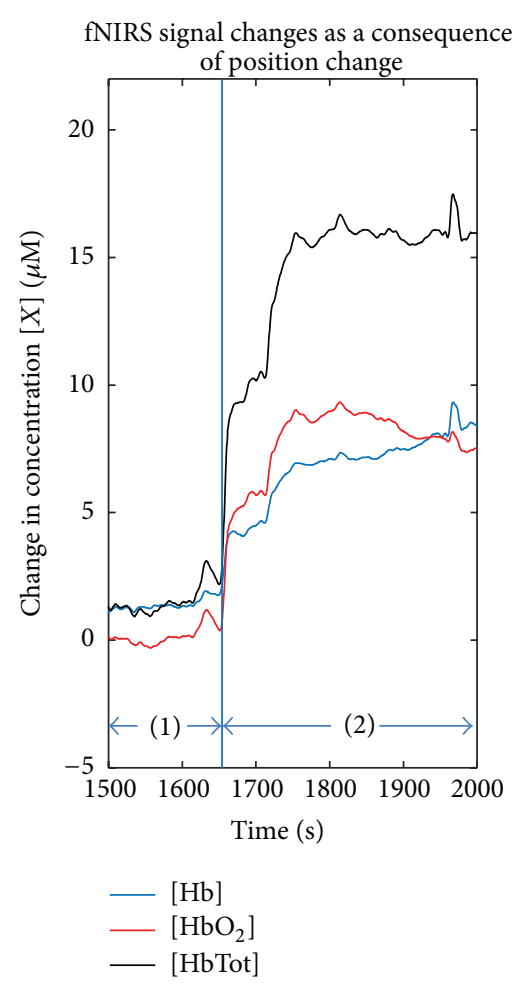

(a)

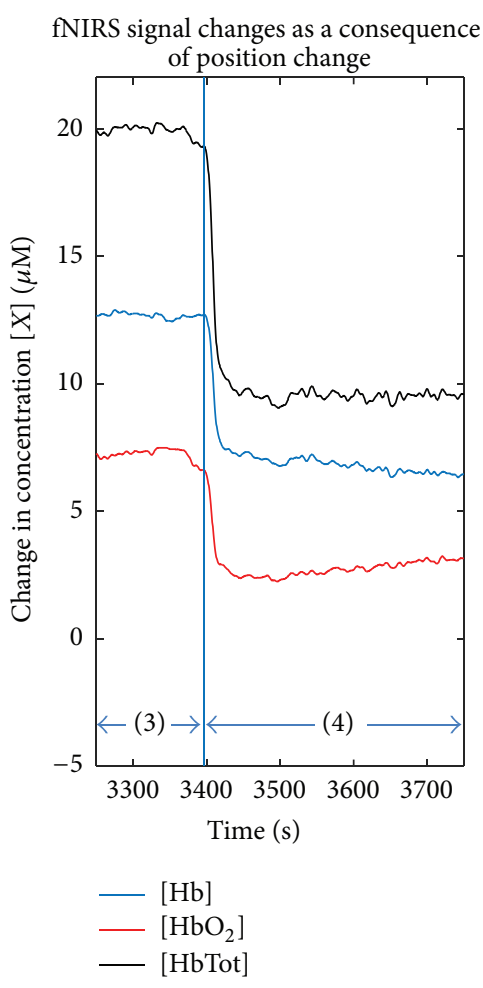

(b)

FIGURE 2: Example of the effect of changes in position on female patient (58 years old) during laparoscopic salpingo-oophorectomy using general anesthesia with $2.4 \%$ sevoflurane. (a) Response of $\mathrm{Hb}, \mathrm{HbO}_{2}$, and $\mathrm{HbTot}$ during transition from (1) supine position to (2) Trendelenburg position. (b) Transition from (3) Trendelenburg position to (4) supine position. (G. Hernandez-Meza, personal communications, July 22, 2015, School of Biomedical Engineering, Science and Health Systems at Drexel University, Philadelphia, PA 19104).

propofol and desflurane groups, with propofol experiencing a larger decrease [43]. Similarly, movement from supine to beach chair position (BCP) was associated with a $10 \%$ decrease in $\mathrm{rSO}_{2}$ that was reversible after returning to the supine position [44]. Head-up position was also evaluated by Kitajima et al. [45] during maintenance with sevoflurane combined with $66 \% \mathrm{~N}_{2} \mathrm{O}$. After changing to the headup position, $\mathrm{HbO}_{2}$ decreased significantly and continued to decrease for 30 minutes after the movement [45]. The recorded $\mathrm{HbO}_{2}$ values remained lower than the baseline even after returning to supine position [45]. A similar effect was observed in HbTot [45]. In contrast, $\mathrm{Hb}$ did not experience significant changes with the movement [45].

Lovell et al. [46] examined changes in blood volume with changes in position during propofol anesthesia. These investigators found increases in CBV during the headdown position that correlated with the degree of table tilt. The degree of table tilt for the head-up position was also correlated with table tilt angle, but the changes during headup position were markedly lower than the equivalent degree of change in the head-down position [46].

The studies outlined in this section demonstrate that changes in position can have significant effect on fNIRS derived measures such as $\mathrm{rSO}_{2}, \mathrm{HbTot}, \mathrm{HbO}_{2}$, and $\mathrm{Hb}$. The changes that occur depend on the type of position and the anesthetics in use. We have observed such positional effects on hemodynamic parameters as measured by fNIRS in our prior study. Figure 2(a) presents an example of the change that is observed on the hemodynamic parameters as a result of a transition from supine to Trendelenburg position. Figure 2(b) shows an example of a return to supine position from Trendelenburg position.

3.2.2. Effects of Other Confounders. The partial pressure of carbon dioxide $\left(\mathrm{PaCO}_{2}\right)$ dissolved in the blood can affect the cerebral blood volume. Low levels of carbon dioxide $\left(\mathrm{CO}_{2}\right)$ will cause a reduction in $\mathrm{CBF}$. Alterations in intrathoracic pressure, such as during $\mathrm{CO}_{2}$ insufflation for laparoscopic procedures, can cause alterations in cerebral perfusion and $\mathrm{PaCO}_{2}$. A study by Owen-Reece et al. [47] showed that CBV, as measured by fNIRS, falls when the intrathoracic pressure is increased. Hyperventilation to decrease $\mathrm{PaCO}_{2}$ also led to a significant decrease in $\mathrm{rSO}_{2}$ in groups of propofolremifentanil and sevoflurane anesthesia [48]. The evaluation by Kim et al. [49] focusing on the effect of preoxygenation with $100 \%$ oxygen found that administration of propofol with sufentanil or midazolam after preoxygenation did not have an effect on $\mathrm{rSO}_{2}$ values.

Another confounding element influencing the fNIRS signal is hemodilution experienced during blood loss, when the lost volume is replaced by crystalloid leading to a decrease in the volume of erythrocytes. This effect was examined 
via measurements of $\mathrm{rSO}_{2}$ during propofol and sevoflurane anesthesia [50]. During the study, oxygen saturation decreases were measured with both anesthetics [50]. Because fNIRS values are dependent on hemoglobin concentration, hemodilution during surgery can influence the hemodynamic parameters measured by fNIRS.

The effects of changes in mean arterial pressure (MAP) have also been evaluated as potential confounders of the fNIRS signal. Nissen et al. [51] evaluated $\mathrm{rSO}_{2}$ during blood pressure decreases that occur after IV administration of propofol and fentanyl during induction. This study found that cerebral oxygenation increased and remained stable during the surgery; however no correlation between MAP and $\mathrm{rSO}_{2}$ could be established [51]. The elevation in $\mathrm{rSO}_{2}$ during induction does however support the trends reported by other authors $[34,35]$.

\subsection{Findings on fNIRS Biomarkers of Depth of Anesthesia.} Initial fNIRS studies examined the hemodynamic effects of anesthetic drugs on $\mathrm{rSO}_{2}, \mathrm{CBV}$, and CBF. More recent studies have focused on finding differences in the $\mathrm{HbO}_{2}, \mathrm{Hb}$, and HbTot biomarkers that could signal transitions between anesthetic states. A summary of the findings of studies included in this section can be found in Table 3. The first study to examine fNIRS biomarkers for depth of anesthesia was performed to validate the capacity of fNIRS to discriminate between "deep" and "light" anesthesia [52]. fNIRS data was collected in the operating room during abdominal or lower body surgical procedures. Deep anesthesia was classified as the 4minute period prior to wound closure, while light anesthesia included the 4 minutes prior to eye opening. The evaluation consisted of a comparison between the levels of $\mathrm{HbO}_{2}, \mathrm{Hb}$, and $\mathrm{HbTot}$ in 26 patients undergoing surgery with general anesthesia using either inhaled sevoflurane or desflurane [52]. The investigators established that across all prefrontal cortex PFC channels there was a statistically significant decrease in the levels of $\mathrm{Hb}$ during the transition from deep to light anesthesia [52]. The most significant changes were found on the right side of the PFC [52]. Similar changes were also observed in our prior study. One example of the changes in the fNIRS signal observed during the transition between maintenance and emergence is presented in Figure 3.

An additional study with a sample size of 20 patients undergoing coloproctology surgery also examined the concentration changes of $\mathrm{Hb}$ in the prefrontal cortex during different stages of anesthesia [53]. In the cases evaluated within this study, propofol was administered to induce anesthesia and sevoflurane was the maintenance agent. The investigators found that the average $\mathrm{Hb}$ concentration was significantly increased from baseline after propofol induction [53]. The comparison of average $\mathrm{Hb}$ concentration between deep and light anesthesia showed a decrease of $\mathrm{Hb}$ as the patient began to wake, and this change reached statistical significance on the right side of the PFC [53].

In a similar manner, fNIRS was used to evaluate the effects of propofol sedation during 41 outpatient elective colonoscopy procedures [54]. Due to previous results, channels located on the right PFC were selected as the PFC areas

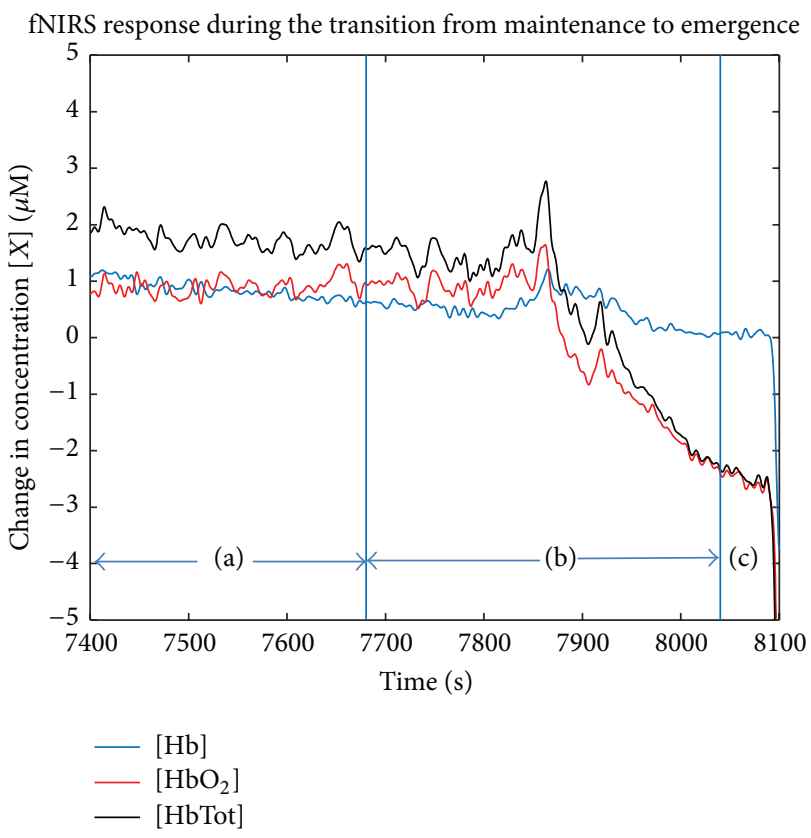

FIGURE 3: Continuous recording of fNIRS signal response during the transition from maintenance to emergence in a 62-year-old male patient. (a) Maintenance with $2 \%$ sevoflurane, (b) anesthetic removal, and (c) response 1 minute before the patient started moving. (G. Hernandez-Meza, personal communications, July 22, 2015, School of Biomedical Engineering, Science and Health Systems at Drexel University, Philadelphia, PA 19104).

of interest and the concentrations of $\mathrm{HbO}_{2}$ and $\mathrm{Hb}$ were analyzed before and after propofol bolus infusions at different concentrations. In this study, a dose dependent increase in $\mathrm{HbO}_{2}$ became significant at the two- and three-minute marks after propofol administration [54]. Significant positive correlation between the change in $\mathrm{HbO}_{2}$ and the administered dose was also found [54]. No significant changes were found in $\mathrm{Hb}$ for propofol sedation doses. These results are in accordance with previous reports showing significant increases in $\mathrm{HbO}_{2}$ following propofol induced anesthesia [34].

\section{Conclusions and Future Directions}

Findings evidencing a reduction of the cerebral metabolic rate of oxygen as a consequence of anesthetic use in regions that included the prefrontal cortex served as motivation for the study of the sensitivity of the hemodynamic response, as measured by fNIRS, as a monitor of depth of anesthesia. Early work investigated metabolic and hemodynamic differences between anesthetics, indicating that fNIRS was capable of measuring the small changes in hemoglobin concentration that occurred in response to different anesthetics and concentrations. Although the literature on the subject is limited, fNIRS has been shown to provide information on cerebral oxygenation that is discriminative between anesthetic states [34-36, 38, 52-54], concentrations of the same anesthetic $[36,38,52-54]$, and different anesthetics $[34,36]$. 
Because the hemodynamic response measured by fNIRS is only capable of providing information on the hemoglobin species concentration it is an indirect assessment of the metabolic rate. As such, it can be affected by confounders that influence the relative quantities of $\mathrm{Hb}$ and $\mathrm{HbO}_{2}$ without changes in the metabolic rate. Position [42-46], systemic $\mathrm{CO}_{2}$ and $\mathrm{O}_{2}$ concentration [47-49], and hemodilution [50] are some of the possible confounders. Careful examination is needed to determine the hemodynamic relationships that are least affected by confounding factors or algorithms will need to be developed to mitigate their effects. Some investigators have already begun to devise algorithms to rule out possible confounders on the NIRS signal during routine oxygen saturation monitoring [55]; however, these algorithms have not yet been integrated into the monitoring software.

In the available literature only three studies were found that specifically investigated biomarkers for depth of anesthesia using the fNIRS signal. The studies by Izzetoglu et al. [52] and Leon-Dominguez et al. [53] found significant changes in $\mathrm{Hb}$ as the subject transitioned between deep and light anesthetic states during the use of sevoflurane and desflurane. The study by Curtin et al. [54] found that propofol prompted a dose dependent increase of $\mathrm{HbO}_{2}$. These findings suggest that fNIRS measured biomarkers such as $\mathrm{Hb}$ and $\mathrm{HbO}_{2}$ concentrations on the prefrontal cortex are sensitive to the effects of different anesthetic types and concentrations [5254]. In addition, fNIRS is sensitive to different anesthetics such as propofol, sevoflurane, and desflurane at sedation and surgical doses [52-54]. Further research is needed to determine if other features of the fNIRS signal contain reliable information on the anesthetic depth. Future efforts should focus on the development of real time examination methods to assess the capacity of the fNIRS signal to provide reliable information that can be used to guide anesthesia delivery. Altogether, with its portability, affordability, safety, ease of use, and the current findings suggesting that fNIRS is indeed suitable for measuring the effects of anesthetics on the suppression of awareness and its return, this makes fNIRS attractive for further research in the field of depth of anesthesia monitoring.

\section{Conflict of Interests}

The authors declare that there is no conflict of interests regarding the publication of this paper.

\section{References}

[1] P. S. Sebel, T. A. Bowdle, M. M. Ghoneim et al., "The incidence of awareness during anesthesia: a multicenter United States study," Anesthesia \& Analgesia, vol. 99, no. 3, pp. 833-839, 2004.

[2] R. H. Sandin, G. Enlund, P. Samuelsson, and C. Lennmarken, "Awareness during anaesthesia: a prospective case study," The Lancet, vol. 355, no. 9205, pp. 707-711, 2000.

[3] G. A. Mashour, A. Shanks, K. K. Tremper et al., "Prevention of intraoperative awareness with explicit recall in an unselected surgical population: a randomized comparative effectiveness trial," Anesthesiology, vol. 117, no. 4, pp. 717-725, 2012.
[4] L. Xu, A.-S. Wu, and Y. Yue, "The incidence of intra-operative awareness during general anesthesia in China: a multi-center observational study," Acta Anaesthesiologica Scandinavica, vol. 53, no. 7, pp. 873-882, 2009.

[5] J. J. Pandit, T. M. Cook, W. R. Jonker, and E. O’Sullivan, “A national survey of anaesthetists (NAP5 Baseline) to estimate an annual incidence of accidental awareness during general anaesthesia in the UK," Anaesthesia, vol. 68, no. 4, pp. 343-353, 2013.

[6] K. Leslie, M. T. V. Chan, P. S. Myles, A. Forbes, and T. J. McCulloch, "Posttraumatic stress disorder in aware patients from the B-aware trial," Anesthesia \& Analgesia, vol. 110, no. 3, pp. 823-828, 2010.

[7] M. T. Alkire, R. J. Haier, S. J. Barker, N. K. Shah, J. C. Wu, and Y. J. Kao, "Cerebral metabolism during propofol anesthesia in humans studied with positron emission tomography," Anesthesiology, vol. 82, no. 2, pp. 393-403, 1995.

[8] M. T. Alkire, R. J. Haier, N. K. Shah, and C. T. Anderson, "Positron emission tomography study of regional cerebral metabolism in humans during isoflurane anesthesia," Anesthesiology, vol. 86, no. 3, pp. 549-557, 1997.

[9] M. T. Alkire, C. J. D. Pomfrett, R. J. Haier et al., "Functional brain imaging during anesthesia in humans: effects of halothane on global and regional cerebral glucose metabolism," Anesthesiology, vol. 90, no. 3, pp. 701-709, 1999.

[10] P. M. Patel and J. C. Drummond, "Cerebral physiology and the effects of anesthetic drugs," in Miller's Anesthesia, vol. 1, pp. 594674, 2010.

[11] U. León-Domínguez, A. Vela-Bueno, M. Froufé-Torres, and J. León-Carrión, "A chronometric functional sub-network in the thalamo-cortical system regulates the flow of neural information necessary for conscious cognitive processes," Neuropsychologia, vol. 51, no. 7, pp. 1336-1349, 2013.

[12] R. A. Veselis, R. A. Reinsel, V. A. Feshchenko, and A. M. Dnistrian, "A neuroanatomical construct for the amnesic effects of propofol," Anesthesiology, vol. 97, no. 2, pp. 329-337, 2002.

[13] E. R. John and L. S. Prichep, "The anesthetic cascade: a theory of how anesthesia suppresses consciousness," Anesthesiology, vol. 102, no. 2, pp. 447-471, 2005.

[14] W. Heinke, C. J. Fiebach, C. Schwarzbauer, M. Meyer, D. Olthoff, and K. Alter, "Sequential effects of propofol on functional brain activation induced by auditory language processing: an event-related functional magnetic resonance imaging study," British Journal of Anaesthesia, vol. 92, no. 5, pp. 641-650, 2004.

[15] American Society of Anesthesiologists (ASA), Standards for Basic Anesthetic Monitoring, ASA House of Delegates, 1986.

[16] M. S. Avidan and G. A. Mashour, "The incidence of intraoperative awareness in the UK: under the rate or under the radar?" British Journal of Anaesthesia, vol. 110, no. 4, pp. 494-497, 2013.

[17] S. C. Manyam, D. K. Gupta, K. B. Johnson et al., "When is a bispectral index of 60 too low? Rational processed electroencephalographic targets are dependent on the sedative-opioid ratio," The Journal of the American Society of Anesthesiologists, vol. 106, no. 3, pp. 472-483, 2007.

[18] R. J. Gajraj, M. Doi, H. Mantzaridis, and G. N. C. Kenny, "Analysis of the EEG bispectrum, auditory evoked potentials and the EEG power spectrum during repeated transitions from consciousness to unconsciousness," British Journal of Anaesthesia, vol. 80, no. 1, pp. 46-52, 1998.

[19] S. von Delius, P. Thies, T. Rieder et al., "Auditory evoked potentials compared with bispectral index for monitoring of 
midazolam and propofol sedation during colonoscopy," The American Journal of Gastroenterology, vol. 104, no. 2, pp. 318325, 2009.

[20] A. Villringer, J. Planck, C. Hock, L. Schleinkofer, and U. Dirnagl, "Near infrared spectroscopy (NIRS): a new tool to study hemodynamic changes during activation of brain function in human adults," Neuroscience Letters, vol. 154, no. 1-2, pp. 101104, 1993.

[21] P. W. McCormick, M. Stewart, M. G. Goetting, M. Dujovny, G. Lewis, and J. I. Ausman, "Noninvasive cerebral optical spectroscopy for monitoring cerebral oxygen delivery and hemodynamics," Critical Care Medicine, vol. 19, no. 1, pp. 89-97, 1991.

[22] A. Villringer and B. Chance, "Non-invasive optical spectroscopy and imaging of human brain function," Trends in Neurosciences, vol. 20, no. 10, pp. 435-442, 1997.

[23] J. C. Rajapakse, F. Kruggel, J. M. Maisog, and D. Y. von Cramon, "Modeling hemodynamic response for analysis of functional MRI time-series," Human Brain Mapping, vol. 6, no. 4, pp. 283300, 1998.

[24] P. J. Magistretti and L. Pellerin, "Cellular mechanisms of brain energy metabolism and their relevance to functional brain imaging," Philosophical Transactions of the Royal Society B: Biological Sciences, vol. 354, no. 1387, pp. 1155-1163, 1999.

[25] P. J. Magistretti, "Cellular bases of functional brain imaging: insights from neuron-glia metabolic coupling," Brain Research, vol. 886, no. 1-2, pp. 108-112, 2000.

[26] X. Cui, S. Bray, D. M. Bryant, G. H. Glover, and A. L. Reiss, "A quantitative comparison of NIRS and fMRI across multiple cognitive tasks," NeuroImage, vol. 54, no. 4, pp. 2808-2821, 2011.

[27] C. Hock, K. Villringer, F. Müller-Spahn et al., "Decrease in parietal cerebral hemoglobin oxygenation during performance of a verbal fluency task in patients with Alzheimer's disease monitored by means of near-infrared spectroscopy (NIRS) correlation with simultaneous rCBF-PET measurements," Brain Research, vol. 755, no. 2, pp. 293-303, 1997.

[28] T. J. Huppert, R. D. Hoge, S. G. Diamond, M. A. Franceschini, and D. A. Boas, "A temporal comparison of BOLD, ASL, and NIRS hemodynamic responses to motor stimuli in adult humans," Neurolmage, vol. 29, no. 2, pp. 368-382, 2006.

[29] J. Lee, B. S. Folley, J. Gore, and S. Park, “Origins of spatial working memory deficits in schizophrenia: an event-related fMRI and near-infrared spectroscopy study," PLoS ONE, vol. 3, no. 3, Article ID e1760, 2008.

[30] B. J. MacIntosh, L. M. Klassen, and R. S. Menon, "Transient hemodynamics during a breath hold challenge in a two part functional imaging study with simultaneous near-infrared spectroscopy in adult humans," NeuroImage, vol. 20, no. 2, pp. 1246-1252, 2003.

[31] C. D. Kurth and W. S. Thayer, "A multiwavelength frequencydomain near-infrared cerebral oximeter," Physics in Medicine and Biology, vol. 44, no. 3, pp. 727-740, 1999.

[32] I. M. Williams, A. J. Picton, S. C. Hardy, A. J. Mortimer, and C. N. McCollum, "Cerebral hypoxia detected by near infrared spectroscopy," Anaesthesia, vol. 49, no. 9, pp. 762-766, 1994.

[33] J. Plachky, S. Hofer, M. Volkmann, E. Martin, H. J. Bardenheuer, and M. A. Weigand, "Regional cerebral oxygen saturation is a sensitive marker of cerebral hypoperfusion during orthotopic liver transplantation," Anesthesia \& Analgesia, vol. 99, no. 2, pp. 344-349, 2004.

[34] A. T. Lovell, H. Owen-Reece, C. E. Elwell, M. Smith, and J. C. Goldstone, "Continuous measurement of cerebral oxygenation by near infrared spectroscopy during induction of anesthesia," Anesthesia \& Analgesia, vol. 88, no. 3, pp. 554-558, 1999.

[35] T. Fleck, S. Schubert, P. Ewert, B. Stiller, N. Nagdyman, and F. Berger, "Propofol effect on cerebral oxygenation in children with congenital heart disease," Pediatric Cardiology, vol. 36, no. 3, pp. 543-549, 2015.

[36] K. Iwasaki, Y. Nomoto, M. Ishiwata, T. Yokota, and R. Ogawa, "Vital capacity induction with $8 \%$ sevoflurane and $\mathrm{N}_{2} \mathrm{O}$ causes cerebral hyperemia," Journal of Anesthesia, vol. 17, no. 1, pp. 3-7, 2003.

[37] L. Valencia, A. Rodríguez-Pérez, B. Kühlmorgen, and R. Y. Santana, "Does sevoflurane preserve regional cerebral oxygen saturation measured by near-infrared spectroscopy better than propofol?" Annales Francaises d'Anesthesie et de Reanimation, vol. 33, no. 4, pp. e59-e65, 2014.

[38] A. Fassoulaki, H. Kaliontzi, G. Petropoulos, and A. Tsaroucha, "The effect of desflurane and sevoflurane on cerebral oximetry under steady-state conditions," Anesthesia \& Analgesia, vol. 102, no. 6, pp. 1830-1835, 2006.

[39] Y. Kanemaru, K. Nishikawa, and F. Goto, "Bispectral index and regional cerebral oxygen saturation during propofol/ $\mathrm{N}_{2} \mathrm{O}$ anesthesia," Canadian Journal of Anesthesia, vol. 53, no. 4, pp. 363-369, 2006.

[40] H. Owen-Reece, C. E. Elwell, W. Harkness et al., "Use of near infrared spectroscopy to estimate cerebral blood flow in conscious and anaesthetized adult subjects," British Journal of Anaesthesia, vol. 76, no. 1, pp. 43-48, 1996.

[41] A. Casati, G. Fanelli, P. Pietropaoli et al., "Monitoring cerebral oxygen saturation in elderly patients undergoing general abdominal surgery: a prospective cohort study," European Journal of Anaesthesiology, vol. 24, no. 1, pp. 59-65, 2007.

[42] S.-J. Kim, J. Y. Kwon, A.-R. Cho, H. K. Kim, and T. K. Kim, "The effects of sevoflurane and propofol anesthesia on cerebral oxygenation in gynecological laparoscopic surgery," Korean Journal of Anesthesiology, vol. 61, no. 3, pp. 225-232, 2011.

[43] J. Y. Kim, J. S. Lee, K. C. Lee, H. S. Kim, S. H. Kim, and H. J. Kwak, "The effect of desflurane versus propofol on regional cerebral oxygenation in the sitting position for shoulder arthroscopy," Journal of Clinical Monitoring and Computing, vol. 28, no. 4, pp. 371-376, 2014.

[44] D. Closhen, M. Berres, C. Werner, K. Engelhard, and P. Schramm, "Influence of beach chair position on cerebral oxygen saturation: a comparison of INVOS and FORE-SIGHT cerebral oximeter," Journal of Neurosurgical Anesthesiology, vol. 25, no. 4, pp. 414-419, 2013.

[45] T. Kitajima, Y. Okuda, S. Yamaguchi, T. Takanishi, M. Kumagai, and K. Ido, "Response of cerebral oxygen metabolism in the head-up position during laparoscopic cholecystectomy," Surgical Laparoscopy \& Endoscopy, vol. 8, no. 6, pp. 449-452, 1998.

[46] T. Lovell, A. C. Marshall, C. E. Elwell, M. Smith, and J. C. Goldstone, "Changes in cerebral blood volume with changes in position in awake and anesthetized subjects," Anesthesia \& Analgesia, vol. 90, no. 2, pp. 372-376, 2000.

[47] H. Owen-Reece, C. E. Elwell, J. Goldstone, M. Smith, D. T. Delpy, and J. S. Wyatt, "Investigation of the effects of hypocapnia upon cerebral haemodynamics in normal volunteers and anaesthetised subjects by near infrared spectroscopy (NIRS)," in Oxygen Transport to Tissue XVI, vol. 361 of Advances in Experimental Medicine and Biology, pp. 475-482, Springer, New York, NY, USA, 1994. 
[48] B. S. Alexander, A. W. Gelb, W. W. Mantulin et al., "Impact of stepwise hyperventilation on cerebral tissue oxygen saturation in anesthetized patients: a mechanistic study," Acta Anaesthesiologica Scandinavica, vol. 57, no. 5, pp. 604-612, 2013.

[49] D. H. Kim, Y. L. Kwak, S. H. Nam, M. S. Kim, E. M. Kim, and J. K. Shim, "Assessment of cerebral oxygen supply-demand balance by near-infrared spectroscopy during induction of anesthesia in patients undergoing coronary artery bypass graft surgery: comparison of midazolam with propofol," Korean Journal of Anesthesiology, vol. 57, no. 4, pp. 428-433, 2009.

[50] K. Yoshitani, M. Kawaguchi, M. Iwata et al., "Comparison of changes in jugular venous bulb oxygen saturation and cerebral oxygen saturation during variations of haemoglobin concentration under propofol and sevoflurane anaesthesia," British Journal of Anaesthesia, vol. 94, no. 3, pp. 341-346, 2005.

[51] P. Nissen, J. J. Van Lieshout, H. B. Nielsen, and N. H. Secher, "Frontal lobe oxygenation is maintained during hypotension following propofol-fentanyl anesthesia," AANA Journal, vol. 77, no. 4, pp. 271-276, 2009.

[52] K. Izzetoglu, H. Ayaz, A. Merzagora et al., "The evolution of field deployable fNIR spectroscopy from bench to clinical settings," Journal of Innovative Optical Health Sciences, vol. 4, no. 3, pp. 239-250, 2011.

[53] U. Leon-Dominguez, M. Izzetoglu, J. Leon-Carrion et al., "Molecular concentration of deoxyHb in human prefrontal cortex predicts the emergence and suppression of consciousness," NeuroImage, vol. 85, pp. 616-625, 2014.

[54] A. Curtin, K. Izzetoglu, J. Reynolds et al., "Functional nearinfrared spectroscopy for the measurement of propofol effects in conscious sedation during outpatient elective colonoscopy," Neurolmage, vol. 85, pp. 626-636, 2014.

[55] A. Denault, A. Deschamps, and J. M. Murkin, "A proposed algorithm for the intraoperative use of cerebral near-infrared spectroscopy," Seminars in Cardiothoracic and Vascular Anesthesia, vol. 11, no. 4, pp. 274-281, 2007. 


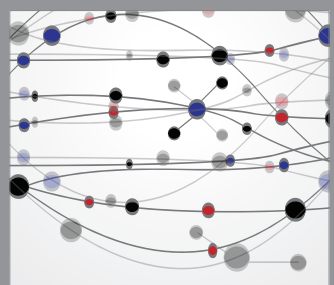

The Scientific World Journal
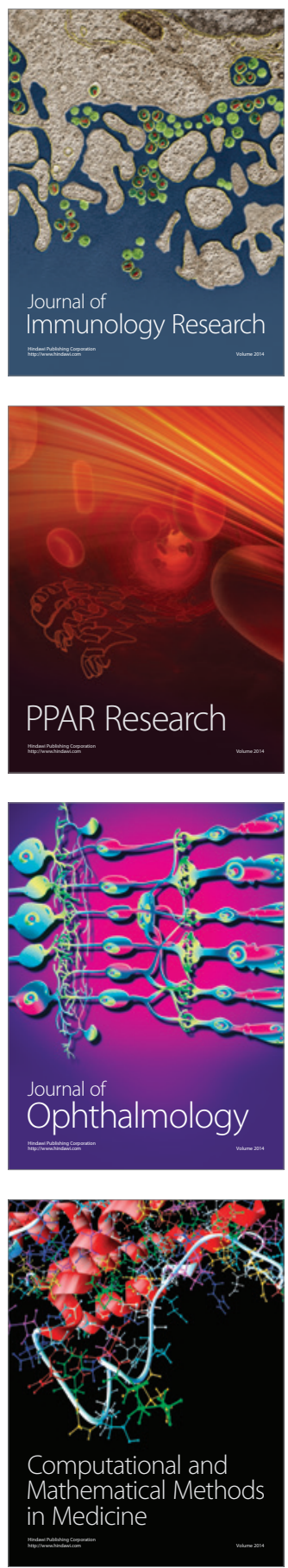

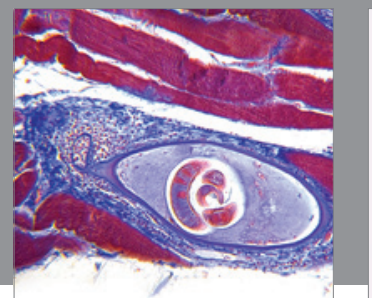

Gastroenterology

Research and Practice
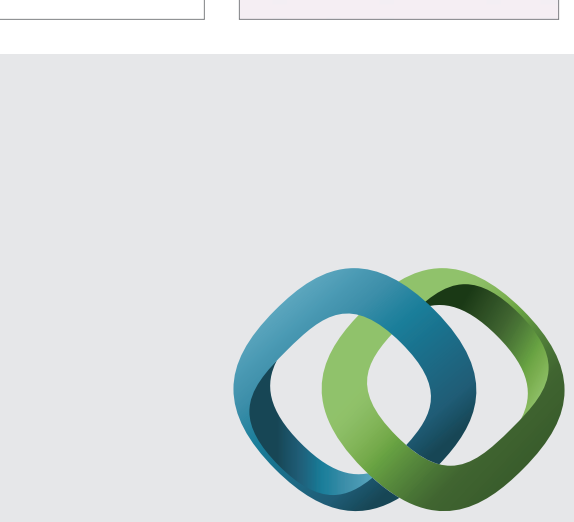

\section{Hindawi}

Submit your manuscripts at

http://www.hindawi.com
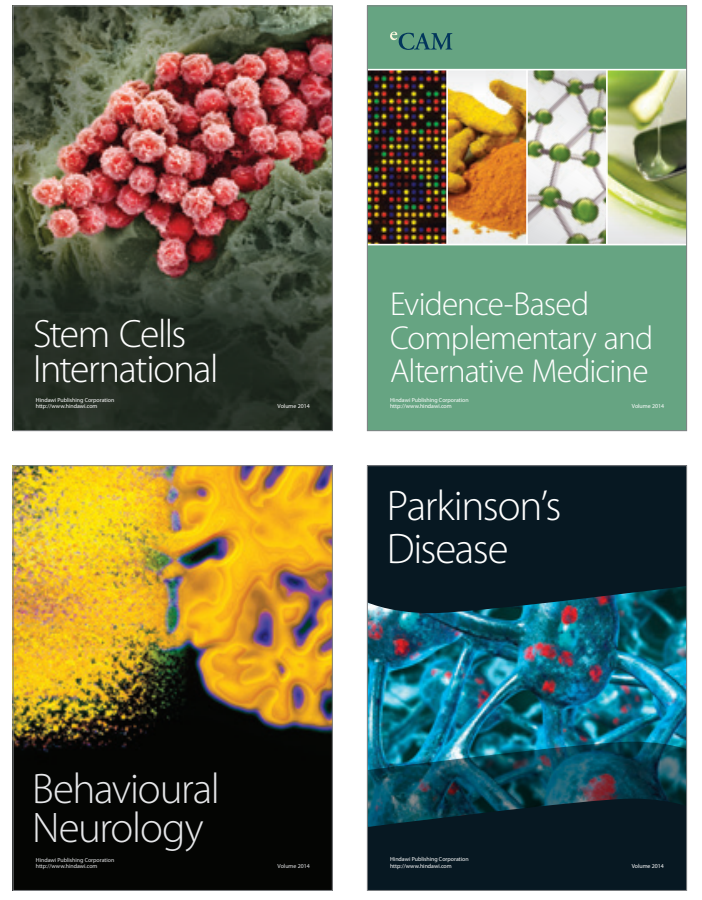
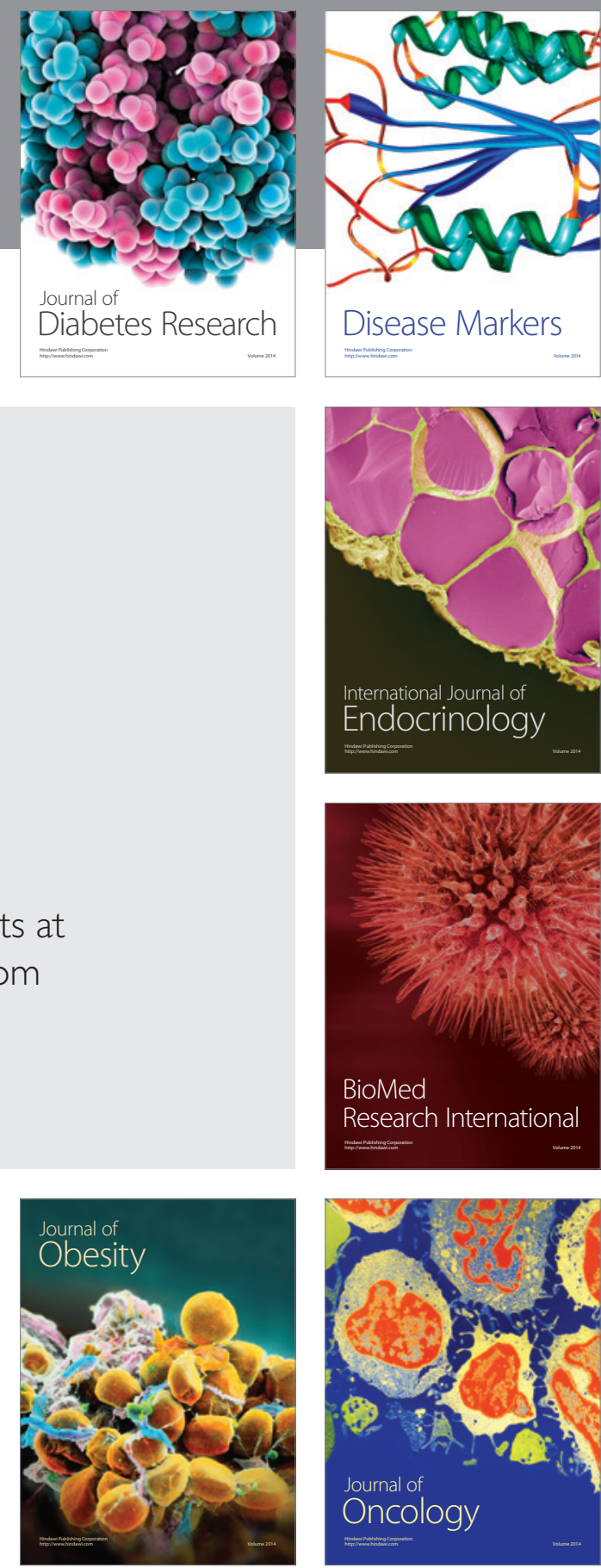

Disease Markers
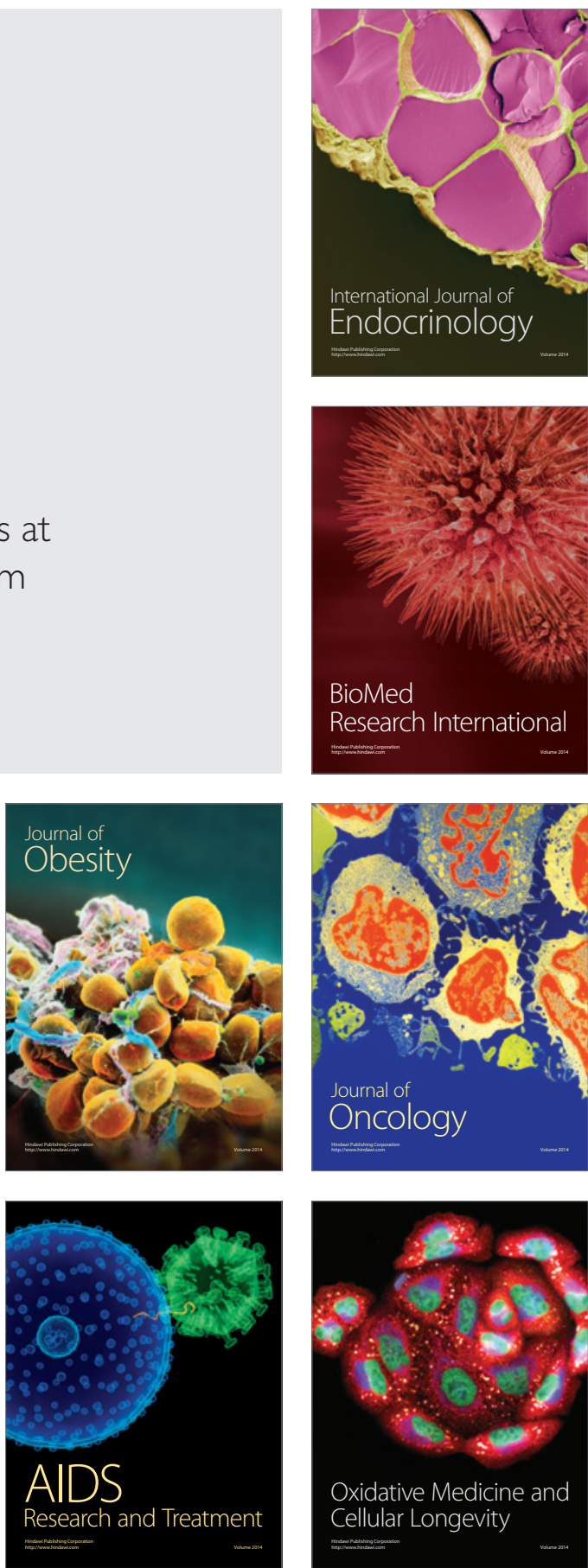